J. Lake Sci.(湖泊科学), 2009, 21(4): 594-602

http://www.jlakes.org. E-mail: jlakes@niglas.ac.cn

(C2009 by Journal of Lake Sciences

\title{
太湖水体秋季散射特性及其相关因素分析*
}

黄昌春, 李云梅, 王 桥, 孙德勇, 乐成峰, 伍 蓝, 王利珍, 王 鍂 (南京师范大学虚拟地理环境教育部重点实验室, 南京 210046)

摘 要: 水体散射特性的研究是水色遥感和水生态学研究中重要的组成部分. 通过对 2007-11-08 至 2007-11-21 在太湖获取的 水体固有光学属性和室内水样分析数据的分析, 研究太湖水体散射特性及其相关因素. 结果表明, 太湖水体散射系数与波长 之间存在幂函数递减规律, 平均幂指数为 $-0.82 \pm 0.21$, 变异系数为 $25.39 \%$; 粒径分布斜率与幂指数以及散射系数 $\ln (550 \mathrm{~nm}) / \ln (756 \mathrm{~nm})$ 之间存在线性相关, $R^{2}$ 分别为 0.894 和 0.783 ; 后向散射率与无机悬浮物浓度之间指数负相关 $R^{2}=0.854$; 折 射指数与无机悬浮物浓度和无机物与有机物的比例之间指数负相关, $R^{2}$ 分别为 0.851 和 0.781 .

关键词: 太湖; 散射特性; 折射指数; 粒径分布斜率

\section{Scattering characteristics and relevant factors in autumn Lake Taihu}

HUANG Chuangchun, LI Yunmei, Wang Qiao, SUN Deyong, LE Chengfeng, WU Lan, WANG Lizhen \& WANG Xing

(Key Laboratory of Virtual Geographic Environment, Ministry of Education, Nanjing Normal University, Nanjing 210046, P.R.China)

\begin{abstract}
Characteristic of water scattering is very important for researches on water color remote sensing and aquatic ecology. The inherent optical properties of water samples collected in Lake Taihu from Nov. 8 to Nov. 21 in 2007 were analyzed, and the scattering characteristics and relative factors were researched. The results showed that, the scattering coefficient was reduced with increasing wavelength according to power function. The average power index is $-0.82 \pm 0.21$, and the variation coefficient is $25.39 \%$. Both slope and power of the particle size distribution function are linearly correlated with the scattering coefficient of $\ln (550 \mathrm{~nm}) / \ln (756 \mathrm{~nm})$. The correlation coefficients $\left(R^{2}\right)$ are 0.894 and 0.783 respectively. Negative correlation between the backscattering rate and the concentration of inorganic suspended was found and $R^{2}$ was 0.854 . Correlations between refractive index and the inorganic suspended solids concentration and between the ratio of inorganic and organic were negative, and the coefficients $\left(R^{2}\right)$ were 0.851 and 0.781 respectively. Keywords: Lake Taihu; scattering characteristics; refractive index; particle size distribution slope
\end{abstract}

水体固有光学属性是水体光学和水色遥感研究的基础，当光子进人到水体中时，大部分的光子被水 体及其组分所吸收，在吸收之前，绝大部分的光子都经历了一次或更多次的散射作用，散射虽然没有使 得可利用的光子量减少(散射的光子仍然可以用于水生植物的光合作用), 但散射使得光线的垂直穿透深 度得到很大的削弱，同时散射作用使得光子通过 Z 型路径从一个颗粒物散射到另外一个颗粒物，极大地 增加光子传输的光学路径, 从而增大了光子被吸收的可能性. 另外, 散射光子中的上行散射光子形成了 上行辐亮度, 上行辐亮度透过水面形成遥感光学信号, 可以说没有散射作用, 就不会有离水辐亮度, 也 就不会有反射率 $[1-2]$. 关于散射系数国内外学者已经做了大量的工作, 纯水散射的研究已经较为成熟并得 到的广泛的应用 ${ }^{[3-4]}$, 水体散射系数随波段的增加存在幂函数递减规律, 但是当浮游色素物质浓度较高时, 散射系数与波段之间的幂函数递减规律减弱甚至不存在 ${ }^{[5-6]}$, 不同水体组分所具有的不同散射相函数对

* “十一五”国家科技支撑计划项目“基于环境一号等国产卫星的大型水体环境遥感监测应用技术与软件研发”(2008BAC34B05) 和江苏省 2008 年度普通高校研究生科研创新计划(CX08B_015Z)联合资助. 2008-08-19 收稿; 2009-01-14 收修改稿. 黄 昌春, 男, 1982 年生, 博士研究生; E-mail: huangchangchun_aaa@163.com. 
水下光场分布和反射率有着较强的影响 ${ }^{[7-8]}$, 因此对水体散射特性的研究有助于利用反射率准确反演水 体组分信息, 有助于对水下光场强度和结构的深人了解, 有助于分析生态光因子对水生态系统的影响.

本研究基于水体固有光学属性测量系统AC-S和BB9实测太湖水体的散射系数和后向散射系数以及 室内实验室分析的水体组分浓度数据, 对水体散射系数特性的影响机理进行分析，进而探讨水体颗粒物 粒径分布、折射指数与散射系数、水体组分浓度之间的相互关系.

\section{1 数据与方法}

\section{1 采样时间及点位}

2007-11-08 至 2007-11-21 对太湖 50 个样点进行了观测和采样, 样点分布如图 1 所示, 测量的参数 包括室内分析的总悬浮物、有机物质、无机物质和叶绿素 $\mathrm{a}$ 的浓度; 野外测量的散射系数和后向散射系 数, 测量深度为 $0.30 \mathrm{~m}$.

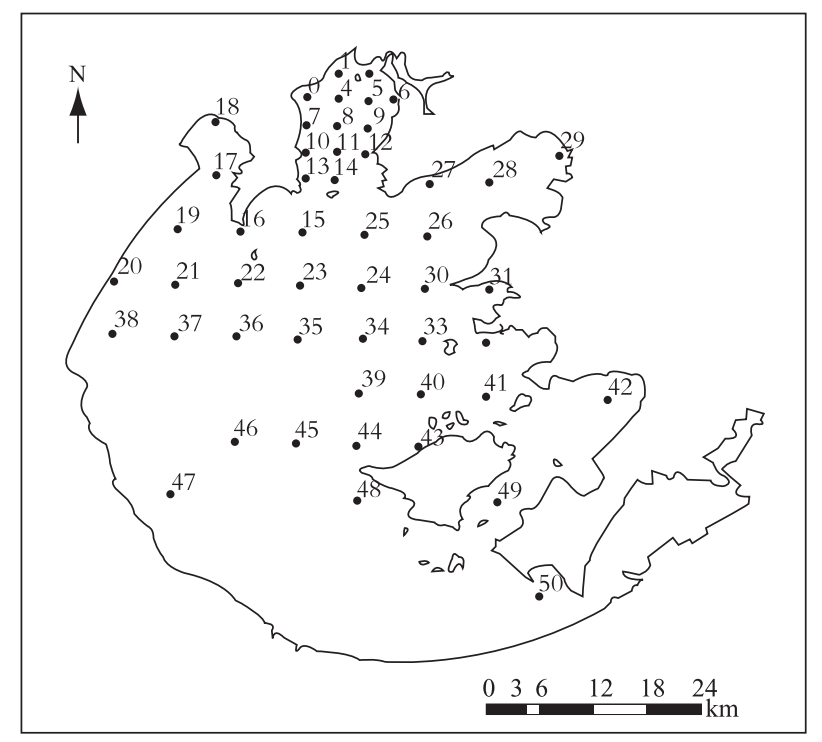

图 1 太湖样点分布

Fig.1 Distribution of sample sites in Lake Taihu

\section{2 参数测定}

1.2.1 散射系数和后向散射系数的测量 散射系数和后向散射系数的测量仪器为 Wetlabs 公司的光谱吸收、 衰减仪(AC-S)和后向散射计 BB9, AC-S 在可见光波长范围共有 85 个光谱通道, 光谱分辨率为 $4 \mathrm{~nm}$, 测量 精度为 $0.01 \mathrm{~m}^{-1}$. 通过 AC-S 可直接获得吸收和衰减系数, 对直接测得的吸收系数作温度、盐度和散射纠 正校正后, 利用衰减系数减去吸收系数得到散射系数 ${ }^{[9]}$; BB9 测得的 $117^{\circ}$ 体散射函数, 利用经验关系计算 得到后向散射系数, 具体处理过程参见文献[9].

1.2.2 CDOM吸收系数的测量 使用 $0.22 \mu \mathrm{m}$ 的滤膜过滤水样, 提取出黄质, 然后, 将黄质溶液装人 $1 \mathrm{~cm} \times 4 \mathrm{~cm}$ 的比色具, 以超纯水作为参考水样, 利用UV-240IPC型紫外分光光度计测量黄质的吸光度, 光 谱吸收系数计算方法为:

$$
a\left(\lambda^{\prime}\right)=2.303 \times D(\lambda) / r
$$

再进行校正：

$$
a(\lambda)=a\left(\lambda^{\prime}\right)-a(750) \times \lambda / 750
$$

其中, $a(\lambda)$ 为波长 $\lambda$ 的吸收系数 $\left(\mathrm{m}^{-1}\right), a(\lambda)$ 为波长 $\lambda$ 未校正的吸收系数 $\left(\mathrm{m}^{-1}\right), D(\lambda)$ 为吸光度, $r$ 为光程路径 $(\mathrm{m})$. 1.2.3 总悬浮物、有机物、无机物和叶绿素浓度的测量 ${ }^{[10]}$ 采用称重法测量总悬浮物、有机物和非有机物 
浓度, 首先进行烧膜处理, 去除膜上有机质, 然后将烧过的膜进行称重, 得到膜的重量 $\mathrm{G} 1$; 将过滤后的 膜进行烘干并称重, 得到干燥膜和总悬浮物的重量 $\mathrm{G} 2$, 两次膜重的差即为总悬浮物重量; 将称重后干燥 的膜进行 $450^{\circ} \mathrm{C}$ 的 $4-6$ 个小时的烧膜处理后称重, 与 $\mathrm{G} 2$ 相减可计算出无机悬浮物重量, 并可进一步推算出 有机悬浮物重量. 用称重法测得的重量与过滤的水样体积相比计算出各参数的浓度, 数据如表 1 所示.

利用“热乙醇法”测量叶绿素浓度 $C_{p h}{ }^{[11]}$.

表 1 水体组分浓度

Tab. 1 Concentrations of water components

\begin{tabular}{ccccc}
\hline 项目 & 最大值 $(\mathrm{mg} / \mathrm{L})$ & 最小值 $(\mathrm{mg} / \mathrm{L})$ & 平均值 $(\mathrm{mg} / \mathrm{L})$ & 变异系数 $(\%)$ \\
\hline 总悬浮物浓度 & 74.65 & 8.2 & $27.33 \pm 16.15$ & 59.10 \\
有机物质浓度 & 58.66 & 2.6 & $18.84 \pm 16.15$ & 79.34 \\
无机物质浓度 & 41.00 & 1.52 & $9.190 \pm 5.65$ & 61.46 \\
叶绿素浓度 & 0.03306 & 0.00096 & $0.01037 \pm 0.00066$ & 63.18 \\
\hline
\end{tabular}

\section{2 结果与分析}

\section{1 散射特征}

太湖水体绝对散射系数较大，在蓝、绿、红波段(波长 $440 、 555 、 675 \mathrm{~nm}$ )最大值分别为 $60.2 、 54.4$ 、 50.2 , 最小值分别为 $5.7 、 4.9 、 4.0$, 远远高于海洋水体的散射系数; 太湖水体散射随波长近似倾斜的直线 或幂函数形式衰减(如图 $2 \mathrm{a}$ 所示), 但是当浮游色素物质较多时, 在 $440 \mathrm{~nm}$ 和 $675 \mathrm{~nm}$ 处出现散射谷值, 这主要 是由于藻类在 $440 \mathrm{~nm}$ 和 $675 \mathrm{~nm}$ 的吸收峰值对散射系数所产生的影响, 然而由于短波段的散射作用相对较强, $675 \mathrm{~nm}$ 相对于 $440 \mathrm{~nm}$ 处的散射谷值要更加明显, 如图 $2 \mathrm{a}$ 中的小图所示, 这使得散射系数随波长单一的直线或 幂函数衰减形式得到衰弱, 甚至不存在. Morel等 ${ }^{[5]}$ 对叶绿素主导的一类水体和黄质主导的二类水体的散射 光谱曲线进行研究认为, 叶绿素主导的一类水体的散射系数与波长之间线性和幂函数关系十分的微弱, 而 黄质主导的二类水体的散射系数与波长之间存在显著的幂函数关系, 幂指数为 -0.6 . Barnard ${ }^{[6]}$ 同样给出了 相应一类水体的平均幂指数为 0.93 . 由于太湖水体组分的差异, 其直线斜率或幂函数指数具有较大的差异, 为了突出散射系数幂指数的明显差异, 对太湖水体散射系数利用公式(3)进行归一化处理:

$$
b_{\text {nor }}=b(\lambda) /<b>
$$
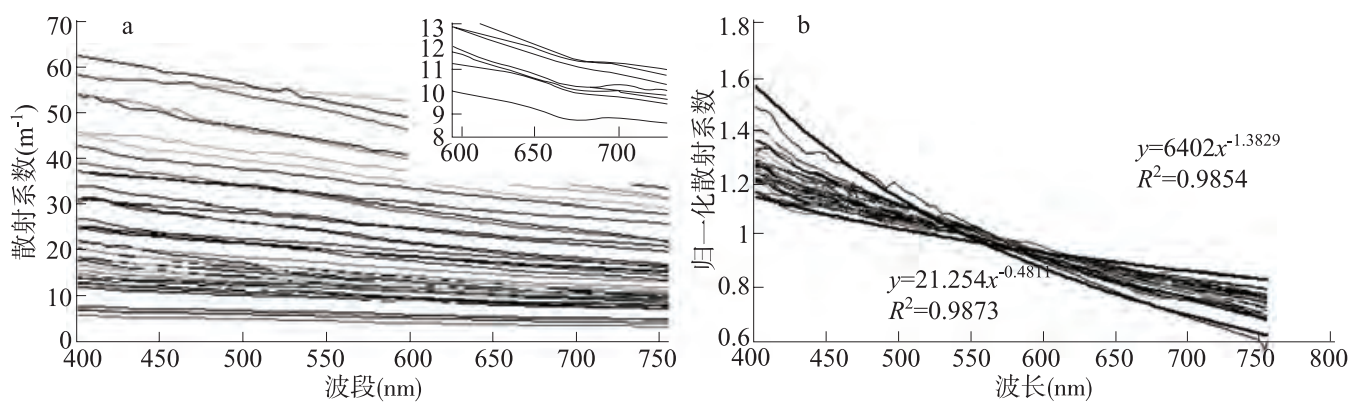

图 2 散射系数与归一化散射系数

Fig.2 Spectral variations of scattering coefficient and normalized scattering coefficient

式中, $b_{\text {nor }}$ 为归一化散射系数, $b(\lambda)$ 为波长为 $\lambda$ 的散射系数, $<b>$ 为一个样点 85 个波段的平均散射系数. 如 图 $2 \mathrm{~b}$ 所示, 太湖水体散射系数与波长之间幂函数平均幂指数为 $-0.82 \pm 0.21$, 变异系数为 $25.39 \%$, 可以 看出太湖水体的幂指数明显要大于黄质主导的二类水体的 0.6 , 而 Barnard 的 0.93 介于本研究幂指数的 范围之内. 
利用平均散射系数 $<b>$ 与每一个波段的散射系数进行相关性分析和线性回归所示, 发现在统计分析 上, 散射系数不存在相关性特别强的优先波段, 其平均相关性 $R^{2}=0.996$, 平均直线斜率为 $1.00 \pm 0.13$, 因 而选用吸收系数较弱的 $550 \mathrm{~nm}$ 波段作为代表波段对散射系数进行研究.

\section{2 比散射系数特征}

散射系数除以总悬浮物质的浓度得到比散射系数如图3所示, 计算公式为:

$$
b^{\mathrm{m}}(\lambda)=b(\lambda) / S P M=b(\lambda) /\left(S P M_{\mathrm{o}}+S P M_{\mathrm{no}}\right)
$$

式中, $b^{\mathrm{m}}(\lambda)$ 为波长为 $\lambda$ 的比散射系数, $S P M$ 为总悬浮物浓度, $S P M_{\mathrm{o}}$ 为有机物的浓度, $S P M_{\mathrm{no}}$ 为无机物的浓 度, $S P M=S P M_{\mathrm{o}}+S P M_{\mathrm{no}}$. 散射系数与总悬浮物浓度和无机物浓度之间具有显著的线性关系, 如图4所示, 相关性分别为 $R^{2}=0.9543$ 和 $R^{2}=0.9225$; 而与有机质之间相关性较弱, 与叶绿素浓度之间几乎不存在相 关性, $R^{2}$ 分别为 0.2518 和 0.0408 . 在大洋一类水体中，有机物的浓度与叶绿素浓度之间具有显著的相关 性 ${ }^{[12-14]}$, 在太湖水体中, 有机物质的浓度与叶绿素浓度之间同样存在一定的线性关系，但是相关性较 低, $R^{2}=0.453$.

理论上，散射系数 $b(\lambda)$ 与总颗粒物体积浓度(总颗粒物体积与水样体积的比例)能够清晰地表示粒径 的分布和折射指数，因而通常利用 $b(\lambda)$ 与总颗粒物体积浓度的比值来刻画颗粒物的特征，而比散射系数 将水体的密度参数和颗粒物的密度参数引人到 $b(\lambda)$ 与总颗粒物体积浓度比值中来, 使得比散射系数的变 化程度远大于 $b(\lambda)$ 与总颗粒物体积浓度的比值.

从散射系数与总悬浮物和无机物浓度之间的关系(图4)可以看出, 如果不考虑总悬浮物中有机物和无 机物的组成比例差异的影响, 比吸收系数 $b^{\mathrm{m}}(\lambda)$ 应该趋向于恒定的值, 然而由于散射系数除受物质的浓度 的影响之外, 还受物质的折射系数 $(n)$ 和粒径分布的影响, 由于有机物质和无机物质的折射系数和粒径分 布存在较大的差异, 从而使得比散射系数并不是恒定值, 对于一类水体 $b^{\mathrm{m}}(555 \mathrm{~nm})$ 的平均值约为 1 , 二类 水体约为 $0.5^{[15]}$, Hofmann ${ }^{[16]}$ 给出了二类水体的 $b^{\mathrm{m}}(\lambda)$ 在 $0.1-0.8$ 范围之内; 而太湖水体 $b^{\mathrm{m}}(550 \mathrm{~nm})$ 在 0.406-1.271范围内, 平均值为 $0.74 \pm 0.18$, 变异系数为 $24.80 \%$, 该范围明显大于Hofmann的 $0.1-0.8$, 说明 太湖水体具有更大的变异性. 与Babin ${ }^{[15,17]}$ 两次巡航以及 $\operatorname{Stramski}^{[18]}$ 数据相比(表2), 太湖水体总比散射系 数与北海、英吉利海峡较相似, 要明显低于大西洋, 但要高于同是Case 2 水体的地中海, 这主要是由于太 湖水体中有机物的含量要远高于地中海 ${ }^{[17]}$.

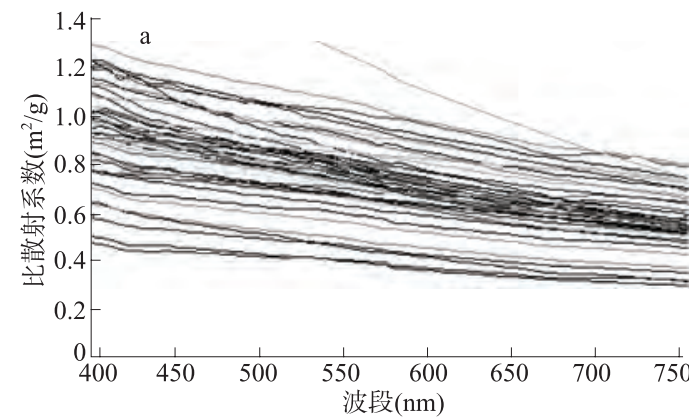

图3 太湖水体比散射系数

Fig.3 Spectral scattering coefficient in Lake Taihu

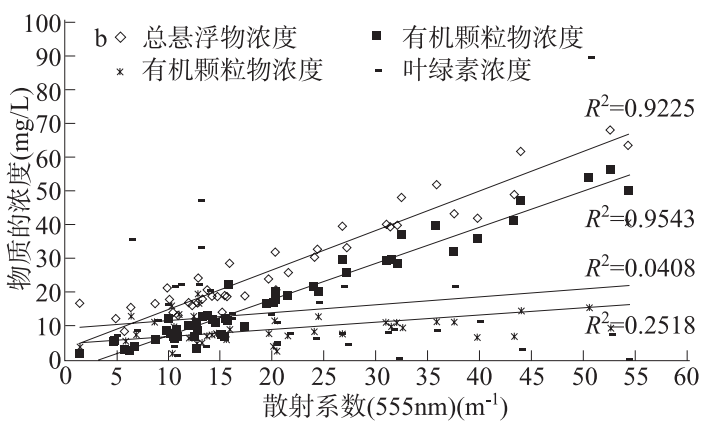

图 $4550 \mathrm{~nm}$ 散射系数与各物质浓度之间的关系 Fig.4 Relationships between the concentrations of substances and scattering coefficient at $550 \mathrm{~nm}$

\section{3 粒径分布}

粒径对散射系数具有强烈的影响, 主要体现在两个方面(1)直接对散射系数的影响, 根据波长和粒径 之间的关系, 散射可以分为瑞利散射和米散射; (2) 由于散射颗粒物并不是非吸收性物质, 吸收系数对散 射的影响随着粒径的增加而迅速增大. 粒径的分布通常具有一定的不规则性, 但是水生态系统中的总悬 浮物质(有机物质、无机物质)一般符合幂函数或双曲线分布方式 ${ }^{[19-21]}$ :

$$
f(D)=k D^{-\xi}
$$




$$
k=(\xi-1) /\left(D_{\min }{ }^{1-\xi}-D_{\max }{ }^{1-\xi}\right)
$$

式中, $D_{\min }$ 为最小粒径下限, $D_{\max }$ 为最大粒径上限, $\xi$ 为粒径分布斜率.

粒径分布斜率 $\xi$ 通过以下步骤计算得到:

(1)颗粒物衰减系数随波长的增加呈指数递减形式 ${ }^{[22]}$ :

$$
c_{\mathrm{p}}(\lambda)=A_{\mathrm{c}} \lambda^{\gamma}
$$

其中, $c_{\mathrm{p}}$ 为颗粒物衰减系数, 通过 $\mathrm{AC}-\mathrm{S}$ 测得的总衰减系数减去 $\mathrm{CDOM}$ 吸收系数得到, $A_{\mathrm{c}}$ 为回归常数, 利 用 $c_{\mathrm{p}}$ 与波长 $\lambda$ 进行指数回归得到其衰减指数 $\gamma$.

(2) Haard ${ }^{[23]}$ 根据Hulst ${ }^{[22]}$ 的mie理论的近似计算方法验证了粒径分布斜率 $\xi$ 与 $\gamma$ 具有如式(8)的线性经验 关系 ${ }^{[23]}$ :

$$
\xi=3-\gamma
$$

Boss $^{[24]}$ 依据最大颗粒物粒径的有限性对其进行了修正, 得到修正公式为:

$$
\xi=3-\gamma-\mathrm{e}^{6 \gamma}
$$

当 $D_{\min }$ 为 $0, D_{\max }$ 为无穷大时, (8)式是准确的, 但是这一情况在显示条件下较难实现, 而(9)式使用范 围为 $1.02 \leqslant n \leqslant 1.2,2.5 \leqslant \xi \leqslant 5$, 因而本研究选用(9)式计算粒径分布斜率, 太湖水体的粒径分布斜率如图 5

\begin{tabular}{|c|c|c|c|c|c|}
\hline Area & 样本数 & 最大值 & 最小值 & 平均值 & S.D \\
\hline 大西洋 ${ }^{[18]}$ & 23 & - & - & 0.985 & 0.402 \\
\hline 波罗的海 ${ }^{[18]}$ & 44 & - & - & 0.571 & 0.356 \\
\hline 英吉利海峡 ${ }^{[18]}$ & 44 & - & - & 0.602 & 0.317 \\
\hline 地中海(Case 2) ${ }^{[18]}$ & 27 & - & - & 0.394 & 0.421 \\
\hline 北海 $^{[18]}$ & 55 & - & - & 0.594 & 0.278 \\
\hline 大西洋[15] & 25 & - & - & 0.97 & $1.9^{*}$ \\
\hline 波罗的海 ${ }^{[15]}$ & 44 & - & - & 0.49 & $1.7^{*}$ \\
\hline 英吉利海峡[15] & 46 & - & - & 0.56 & $1.7^{*}$ \\
\hline 地中海(Case 2) ${ }^{[15]}$ & 35 & - & - & 0.42 & $2.6^{*}$ \\
\hline 北海 ${ }^{[15]}$ & 55 & - & - & 0.54 & $1.6^{*}$ \\
\hline All Case $2^{[15]}$ & 180 & - & - & 0.51 & $1.9^{*}$ \\
\hline 中国土壤 ${ }^{[17]}$ & 4 & 1.17 & 1.02 & - & - \\
\hline 黄海 ${ }^{[17]}$ & 2 & 1.34 & 0.9 & - & - \\
\hline 太湖(本文) & 51 & 1.271 & 0.406 & 0.74 & 0.199 \\
\hline
\end{tabular}
所示.

表 $2550 \mathrm{~nm}$ 比散射系数

Tab.2 Specific scattering coefficient at $550 \mathrm{~nm}$

*为几何标准差.

目前对于粒径分布斜率 $\xi$ 现有的观测值范围在 3-5 $5^{[25]}$ 之间(通过电阻脉冲粒子计数器测量得到), 而活 性颗粒物粒径分布斜率 $\xi$ 范围相对要小约为 3.7-4.3 $3^{[26]}$ (通过显微镜方法测量得到), 太湖水体的 $\xi$ 在现有 观测数据的范围 3-5 之内, 为 3.34-4.54, 其中 3.8-4.4 约占 94\%, 平均值为 $4.05 \pm 0.20$, 变异系数为 $5.03 \%$. 无机物质的散射主要是由小于 $1 \mu \mathrm{m}$ 的无机物质产生的, 约占 $50 \%$ 以上, 而藻类物质的散射主要是由 1-10 $\mu \mathrm{m}$ 范围内藻类物质产生的 ${ }^{[15]}$. 假设折射指数不变, 颗粒物粒径分布符合幂函数形式, 散射系数计算 
公式为:

$$
b_{p}(\lambda)=\frac{\pi}{4} \int_{D \max }^{D \min } f(D) Q_{b}(\lambda, D) D^{2} \mathrm{~d} D
$$

式中, $Q_{b}(\lambda, D)$ 为散射效率因子, 通过 Mie 散射算法 计算得到. 可以看出无机物质和有机物质的比例和 粒径的大小对散射系数的影响十分的显著, 由于 $550 \mathrm{~nm}$ 散射系数受颗粒物和水体吸收系数影响较小, 而 $756 \mathrm{~nm}$ 受颗粒物吸收系数影响较小, 但受水体吸 收影响较强, 同时散射系数与颗粒物浓度之间存在 显著的指数相关性，因而 $\ln (550 \mathrm{~nm}) / \ln (756 \mathrm{~nm})$ 对粒径 分布斜率 $\xi$ 具有一定的指示作用, 经研究发现粒径 分布斜率 $\xi$ 与散射系数 $\ln (550 \mathrm{~nm}) / \ln (756 \mathrm{~nm})$ 之间存 在线性相关关系, $R^{2}=0.7833$, 如图 6a 所示; 散射系数 光谱随波长的增加呈现幂函数衰减, 而粒径的分布 对幂函数指数具有较强的影响, 粒径分布斜率 $\xi$ 与 幂函数指数之间存在的线性关系，如图 $6 \mathrm{~b}$ 所示， $R^{2}=0.8941$.

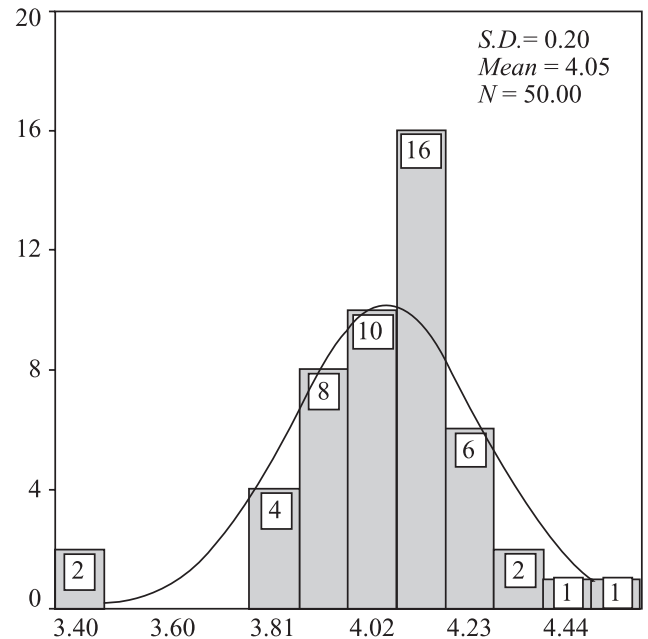

图 5 粒径分布斜率频率图

Fig.5 Frequency distribution of particle size distribution slope
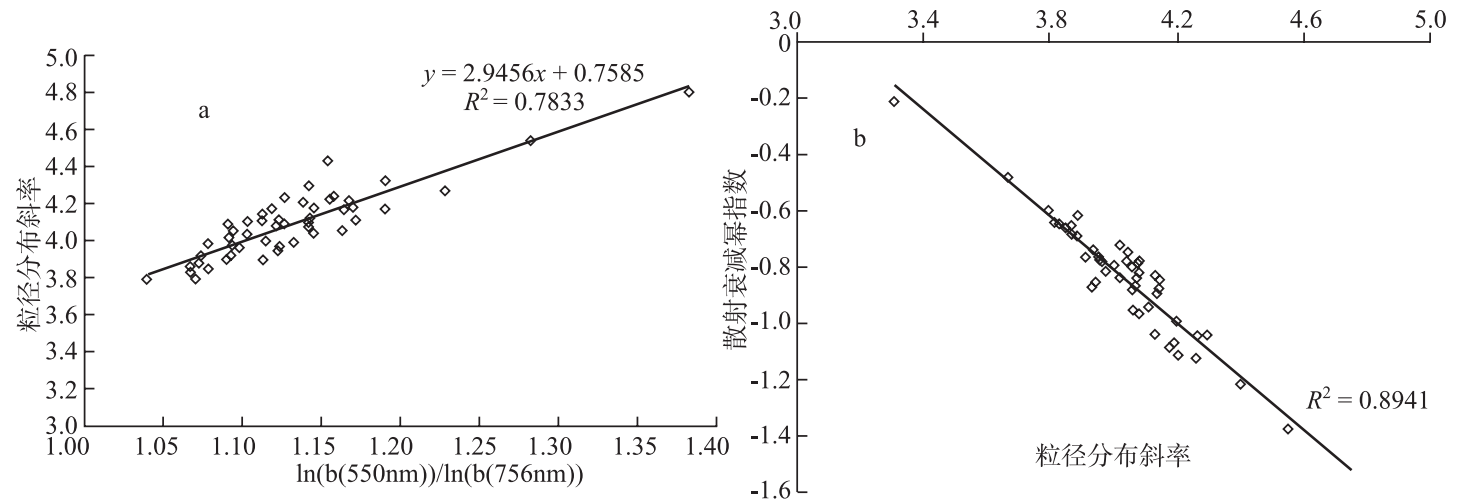

图 6 粒径分布斜率与散射系数之间的关系(a); 粒径分布斜率和散射系数衰减指数之间的关系(b)

Fig.6 The relationship between particle size distribution slope and the scattering coefficient(a), the relationship between particle size distribution slope and scattering coefficient attenuation index(b)

\section{4 折射指数}

根据上述计算出来的 $b_{\mathrm{bp}} / b_{\mathrm{p}}$ 和 $\xi$ 计算折射指数，当 $0<b_{\mathrm{bp}} / b_{\mathrm{p}}<0.03$ 和 $2.5<\xi<4.5$ 时，可以利用如下经 验模型公式计算 ${ }^{[27]}$ :

$$
\mathrm{n}\left(b_{\mathrm{bp}} / b_{\mathrm{p}}, \xi\right)=1+\left(b_{\mathrm{bp}} / b_{\mathrm{p}}\right)^{\left(0.5377+0.4867^{*}(\xi-3)^{2}\right) *\left(1.4676+2.295 *(\xi-3)^{2}+2.3113^{*}(\xi-3)^{4}\right)}
$$

其中 $\mathrm{n}\left(b_{\mathrm{bp}} / b_{\mathrm{p}}, \xi\right)$ 为折射指数，当 $\xi<3.5$ 时，可采用 $(10)$ 式计算折射指数 ${ }^{[7]}$ :

$$
\mathrm{n}\left(b_{\mathrm{bp}} / b_{\mathrm{p}}\right)=1+1.671 *\left(b_{\mathrm{bp}} / b_{\mathrm{p}}\right)^{0.582}
$$

由于本研究的 $\xi$ 的范围为: $3.0<\xi<4.5$, 后向散射率范围为: $0<b_{\mathrm{bp}} / b_{\mathrm{p}}<0.03$, 因而选用式(9)计算折射指数, 太湖水体的折射指数频率分布如图7所示, 范围为1.062912-1.25, 但大部分的折射指数集中在1.07-1.17 范围内, 约占到了 $96 \%$, 平均值为 $1.12111 \pm 0.03227$, 变异系数为 $0.02879 . \mathrm{Aas}^{[28]}$ 认为藻类和泥沙的折射指 
数通常为 1.05 和 1.18 , Twardowski ${ }^{[27]}$ 给出藻类细胞折射指数约为 $1.04-1.05$, 有机非生命物质为 1.05 , 无机

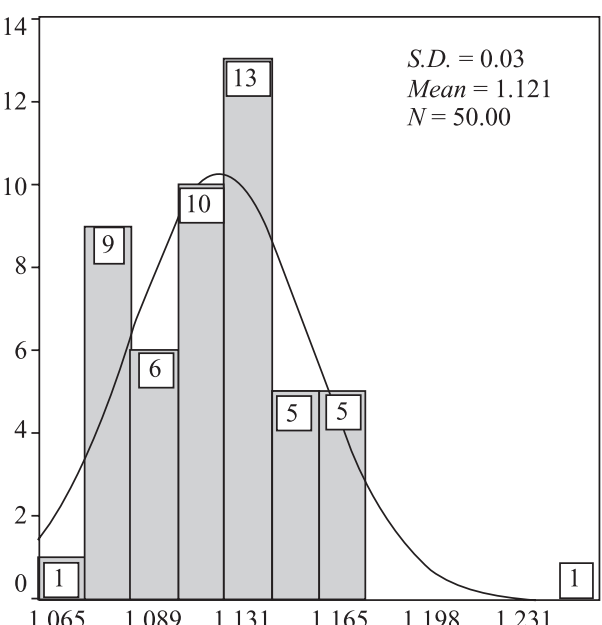

图 7 折射指数频率图

Fig.7 Frequency map of refractive index 非生命物质为 1.14-1.18, Jerlov ${ }^{[29]}$ 对折射指数研究认为, 活性浮游植物折射指数为 1.01-1.09, 碎石和无机颗粒物 为 1.15-1.20, Mobley ${ }^{[30]}$ 认为浮游植物的折射指数约为 1.05 , 无机颗粒物为 1.16 . 因而可以根据折射指数对水体的藻类 和泥沙的组成进行判断 (1)当折射指数 $\leqslant 1.09$ 时浮游藻类 占主导地位(2)当折射指数 $1.09 \leqslant n \leqslant 1.15$ 藻类和泥沙共同 作用(3)当折射指数 $1.15 \leqslant n$ 时, 泥沙占主导地位 ${ }^{[31]}$. 即折 射指数随着无机物浓度的比例增加而增加.

但是太湖水体中无机物和有机物的组成对折射系数 的影响却存在很大的差别, 折射指数并不是随着无机物 浓度的比例增加而增加如图8a所示，随着总悬浮物和无 机物浓度的增加, 折射指数呈现指数形式下降, 相关性 分别达到了 0.8262 和 0.8508 , 而有机物的浓度和叶绿素 的浓度与折射指数的相关性较小, 分别为 0.1423 和 0.049 ; 折射指数与无机物和有机物的比例同样存在较为显著的 相关性，如图 8b所示，随着无机物比例的增加，折射指 数呈指数形式逐渐减小, $R^{2}=0.7807$. 单就数据来看, 这一结果与现有折射指数随着无机物浓度的比例 增加而增加的规律表现出相反的变化趋势，这一现象同时出现在Loisel和Lubac对英吉利海峡固有光学 属性的研究中 ${ }^{[32-33]}$.
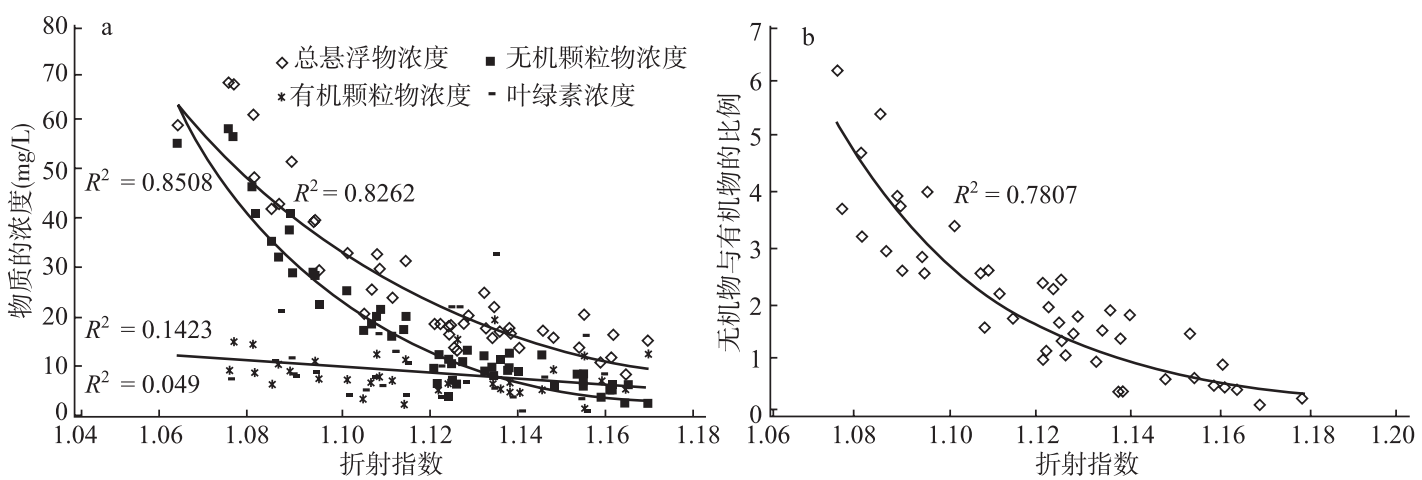

图 8 折射指数与物质的浓度(a)和无机物与有机物比例(b)之间的关系

Fig. 8 Relationship between the refractive index and material concentration(a) and the proportion of organic and inorganic(b)

\section{3 讨论}

折射指数与后向散射率之间存在正相关关系，与无机物浓度之间存在负相关关系，因而通过后向散 射率中的后向散射系数和散射系数这两个因子与无机物浓度之间的关系来分析折射指数与无机物浓度之 间负相关性存在的可能性. 由图 4 可知，散射系数与无机物浓度之间存在较好的线性相关性，随着无机 物浓度的增加, 散射系数呈现线性增长, $R^{2}=0.9543$, 直线斜率为 1.158 ; 后向散射系数与无机物浓度之间 同样存在正相关关系，如图 9a 所示，当后向散射系数 $b_{\mathrm{b}}<0.21$ 时，随着无机物浓度的增加，后向散射系 数呈线性增加, $R^{2}=0.8167$, 直线斜率为 0.0122 , 当后向散射系数 $b_{\mathrm{b}} \geqslant 0.21$ 时, 随着无机物浓度的迅速增加, 后向散射系数呈线性缓慢增加, $R^{2}=0.8464$, 直线斜率为 0.0005 ; 因而他们的比值后向散射率随着无机物 浓度的增加呈现指数形式衰减, 如图 $9 \mathrm{~b}$ 所示, $R^{2}=0.8544$, 从而使得与后向散射率成正相关的折射指数随 
着无机物的浓度的增加呈衰减状态如图 8a.
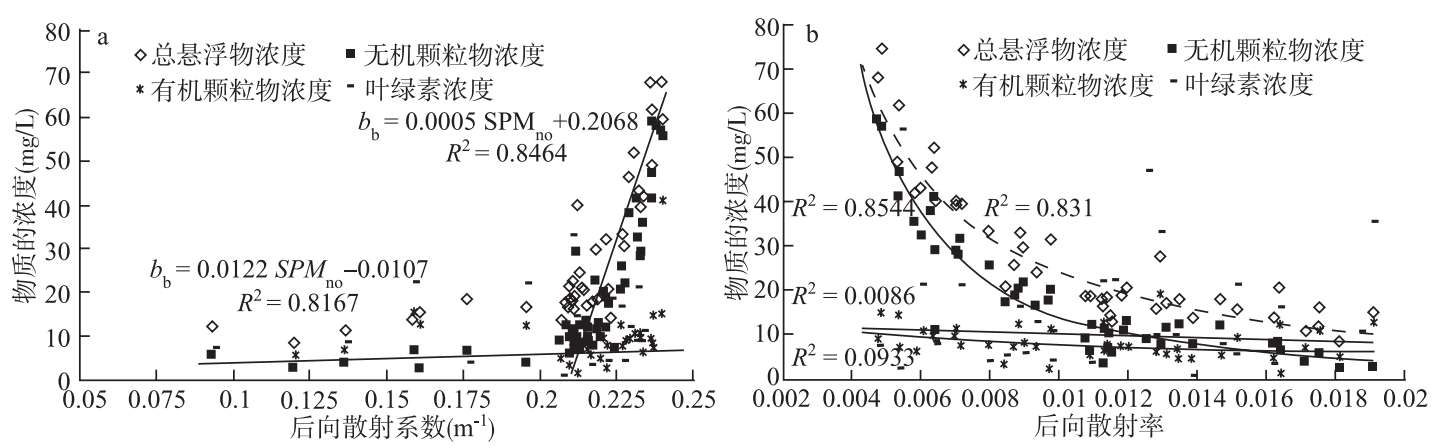

图 9 后向散射系数(a)和后向散射率(b)与物质的浓度之间的关系

Fig.9 Relationship between the backscattering coefficient(a) and backscattering rate(b) and the concentration of substance

随着物质浓度的增加, 小颗粒物质不断吸附 富集，颗粒物粒径逐渐增大，通过 Mie 理论计算后 向散射效率因子, 如图 10 所示, 随着粒径的增加, 后向散射效率总体呈现先增加后减小最终趋向于 一恒定值, 不再随粒径的增加而增加的变化趋势, 说明当颗粒物粒径达到一定数值后, 后向散射系 数随着颗粒物粒径增加趋向于恒定值, 即后向散 射系数随着水体组分浓度的增加趋向于恒定值.

\section{4 结论}

(1) 太湖水体绝对散射系数较大, 散射系数 光谱特征随波长的增加呈现幂函数形式单调递减, 幂指数平均值为 $-0.82027 \pm 0.20823$, 但是在叶绿

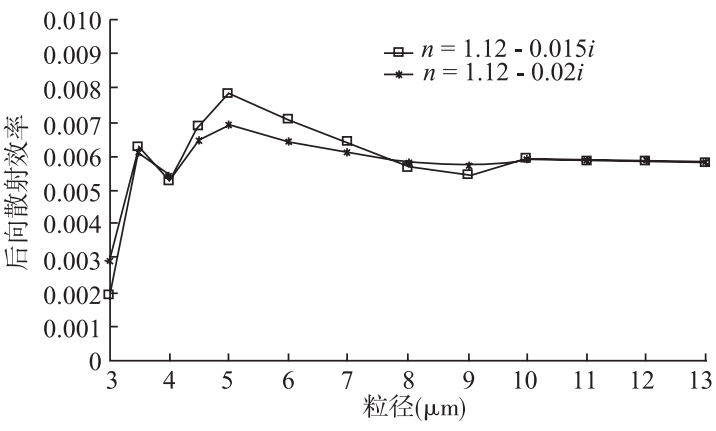

图 10 后向散射效率随粒径的变化

Fig.10 Backscattering efficiency change with the particle size 素浓度较高的地方, 散射系数在 $675 \mathrm{~nm}$ 存在散射谷值, 这一谷值在一定的程度上破坏了散射系数随波长 幂函数递减的规律.

(2) 太湖水体比散射系数 $b^{m}(550 \mathrm{~nm})$ 变化范围为 $0.160066-1.263727$, 平均值为 $0.74093 \pm 0.18373$, 变 异系数为 0.24798 , 跨越了一类水体和二类水体的平均值, 具有更大的复杂性质.

(3) 粒径分布斜率与散射系数衰减指数具有显著的线性相关性, $R^{2}=0.8941$, 与散射系数 $\ln (550 \mathrm{~nm}) / \ln (756 \mathrm{~nm})$ 线性相关, $R^{2}=0.7833$.

(4) 由于散射系数随无机物浓度增加的速度远大于后向散射系数随无机物浓度增加的速度, 从而后 向散射率随无机物浓度的增加而减小, 使得太湖水体折射指数与无机物或有机物浓度成负相关关系.

\section{5 参考文献}

[1] Kirk JTO. Light and photosynthesis in aquatic ecosystems. Cambridge University Press, 1983: 71.

[2] 徐希孺. 遥感物理. 北京: 北京大学出版社, 2005.

[3] Sogandares FM, Fry ES. Absorption spectrum(340-640nm)of pure water I Photo thermal measurements. Applied Optics, 1997, 36(33): 8699-8709.

[4] Pope RM, Fry ES. Absorption spectrum (380-700nm) of pure water. II. Integrating cavitymeasurements. Applied Optics, 1997, 36: $8710-8723$. 
[5] Morel_A, Bernard G, Malik C. Bio-optical properties of high chlorophyll Case 1 waters and of yellow-substance-dominated Case 2 waters. Deep-Sea Research, 2006(doi:10.1016/j.dsr.2006.07.007).

[6] Bricaud A, Morel A. Light attenuation and scattering by phytoplanktonic cells: A theoretical modeling. Applied Optics, 1986, 25: $571-580$.

[7] Mobley CD, Sundman LK, Boss E. Phase function effects on oceanic light fields. Applied Optics, 2002, 41(6): 1035-1050.

[8] John T. Kirk O. Volume scattering function, average cosines, and the underwater light field. Limnol Oceanogr, 1991, 36(3): 455-467.

[9] Moore C, Barnard A, Hankins D et al. Spectral absorption and attenuation meter (ac-s) user's guide, revision A. America: WET Labs Inc, 2004: 5-20.

[10] Mueller JL, Fargion GS, Zaneveld RV et al. Ocean optics protocols for satellite ocean color sensor validation. Revision 4. Volume IV. NASA, 2003.

[11] 陈宇炜, 陈开宁, 胡耀辉. 浮游植物叶绿素a测定的“热乙醇法”及其测定误差探讨. 湖泊科学, 2006, 18: 550-552.

[12] Pierre. et Marie Curie Matie re en suspension dans les eaux de mer: repartition, composition chimique, origine et evolution, Laboratoire de Physique et Chimie Marines. Thesis, Universite, 1980: 173.

[13] Raaphoret VW, Malschaert JFP. Ammonium adsorption in superficial North Sea sediments. Cont Shelf Res, 1996,16 1415-1435.

[14] Morel A. Optical modeling of the upper ocean in relation to its biogenous matter content (Case I waters). Geophys Res, 1988, 93: $10749-10768$.

[15] Babin M, Morel A, Fell F. Light scattering properties of marine particles in coastal and open ocean waters as related to the particle mass concentration. Limnol Oceanogr, 2003, 48(2): 843-859.

[16] Hofmann A, Dominik J. Turbidity and mass concentration of suspended matter in lake water: A comparison of two calibration methods. Aquatic Science, 1995, 57: 54-69.

[17] Stramski1 D, Sławomir B, Flatau WJ. Optical properties of Asian mineral dust suspended in seawater. Limnol Oceanogr, 2004, 49(3): 749-755.

[18] Babin M, Morel A, Fournier-Sicre V et al. Scattering properties of case 2 water particles. Conference proceeding, 2002 : $18-22$.

[19] Bader H. The hyperbolic distribution of particles sizes. Geophys Res, 1970, 75: 2822-2830.

[20] Sheldon RW, Prakash A, Sutcliffe WH. The size distribution of particles in the ocean. Limnol Oceanogr, 1972, 17: $327-340$.

[21] Rodriguez J, Mullin M. Relation between biomass and body weight of plankton in a steady-state oceanic ecosystem. Limnol Oceanogr, 1986, 31: 361-370.

[22] Van de Hulst HC. Light Scattering by Small Particles. New York: John Wiley \& Sons INC, 1957.

[23] Haardt H. Measurement of the spectral attenuation to support biological research in a "plankton tube" experiment. Oceanol, 1980, 3: 89-96.

[24] Boss E, Twardowski MS, Herring S. Shape of the particulate beam attenuation spectrum and its inversion to obtain the shape of the particulate size distribution. Applied Optics, 2001, 40(27): 4885-4893.

[25] Jonasz M. Particle-size distributions in the Baltic. Tellus, 1983, 35: 346-358.

[26] Sprules WG, Munawar M. Plankton size spectra in relation to ecosystem productivity, size, and perturbation. Can J Fish Aquat Sci, 1986, 43: 1789-1794.

[27] Twardowski MS, Boss E, Macdonald JB. Model for estimating bulk refractive index from the optical backscattering ratio and the implications for understanding particle composition in case I and case II waters. Journal of Geophysical Research, 2001, 14: $129-142$.

[28] Aas E. The refractive index of phytoplankton. Department of Geophysics, Oslo Univ, 1981: 61.

[29] Jerlov NG. Marine Optics. New York: Elsevier scientific Pulishing company, 1976.

[30] Mobley CD. Light and water: radiative transfer in natural waters. San Diego: Academic Press, 1994.

[31] 孙德勇, 李云梅, 乐成峰等. 太湖水体散射特性及其与悬浮物浓度关系模型. 环境科学, 2007, 28(12): 2688-2694.

[32] Loisel H, Me'riaux X, Berthon JF. Investigation of the optical backscattering to scattering ratio of marine particles in relation to their biogeochemical composition in the eastern English Channel and southern North Sea. Limnol Oceanogr, 2007, 52(2): 739-752.

[33] Lubac B, Loisel H. Variability and classification of remote sensing reflectance spectra in the eastern English Channel and southern North Sea. Remote Sensing of Environment, 2007, 110: 45-58. 